\title{
Lipid Landscape of the Human Retina and Supporting Tissues Revealed by High Resolution Imaging Mass Spectrometry
}

David M.G. Anderson ${ }^{1}$, Jeffrey D. Messinger ${ }^{2}$, N. Heath Patterson ${ }^{1}$, Emilio Rivera ${ }^{1}$, Jeffrey M. Spraggins $^{1}$, Ankita Kotnala ${ }^{1,2}$, Richard M. Caprioli ${ }^{1}$, Christine A. Curcio ${ }^{2}$, and Kevin L. Schey ${ }^{1}$

${ }^{1}$ Department of Biochemistry, Vanderbilt University, Nashville, TN

${ }^{2}$ Department of Ophthalmology and Visual Sciences, University of Alabama at Birmingham, Birmingham, AL

\section{Supplement Information}

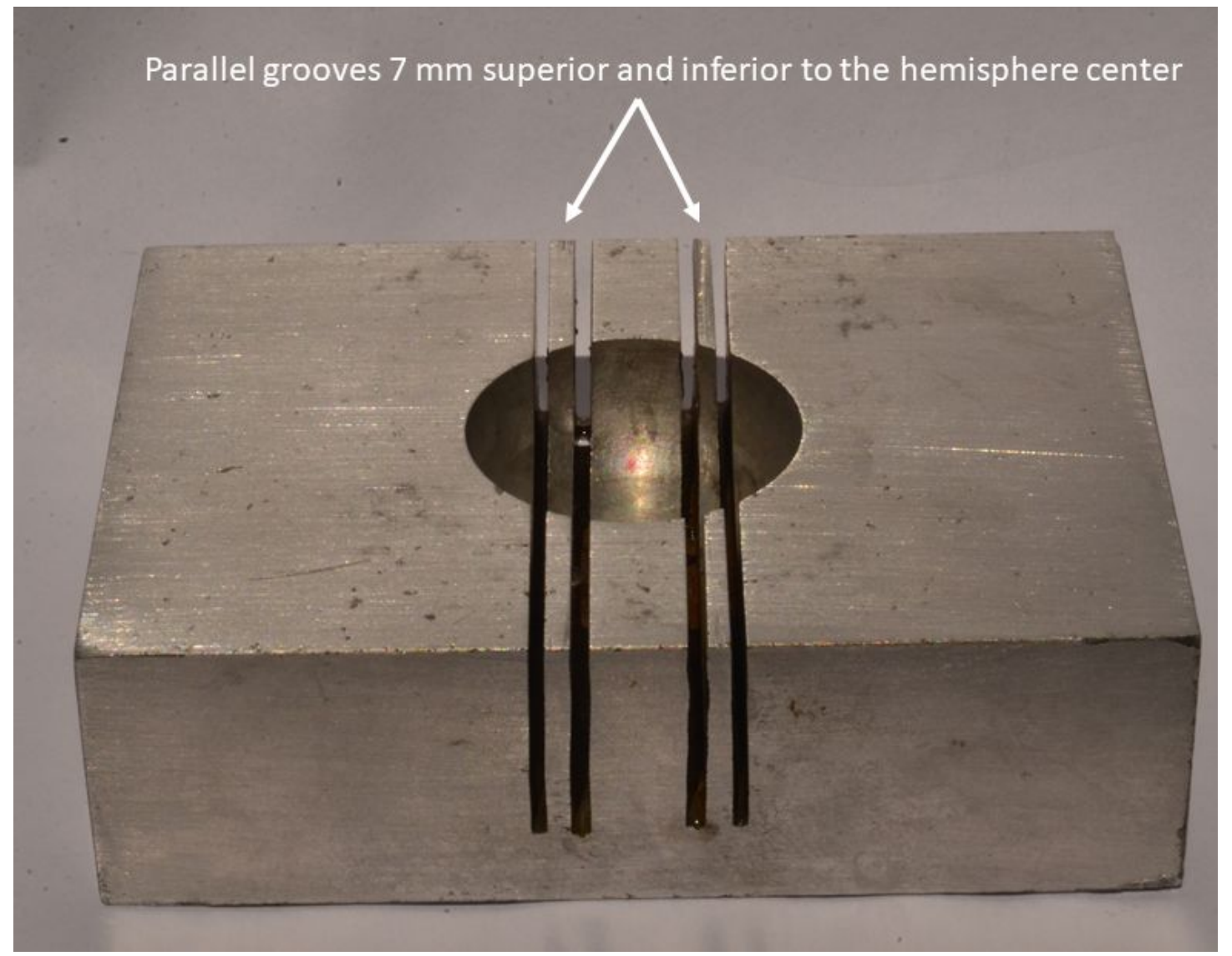

Supplemental Figure 1. Custom-designed aluminum 3" x 4" x 1" billet for standardized dissection of a human eye. A human eye is placed within the $30-\mathrm{mm}$-diameter hemispheric well, looking up. The parallel grooves $7 \mathrm{~mm}$ superior and inferior to the hemisphere center provide orientation of a tissue blade while a tissue belt through the middle of the globe is sliced out. 


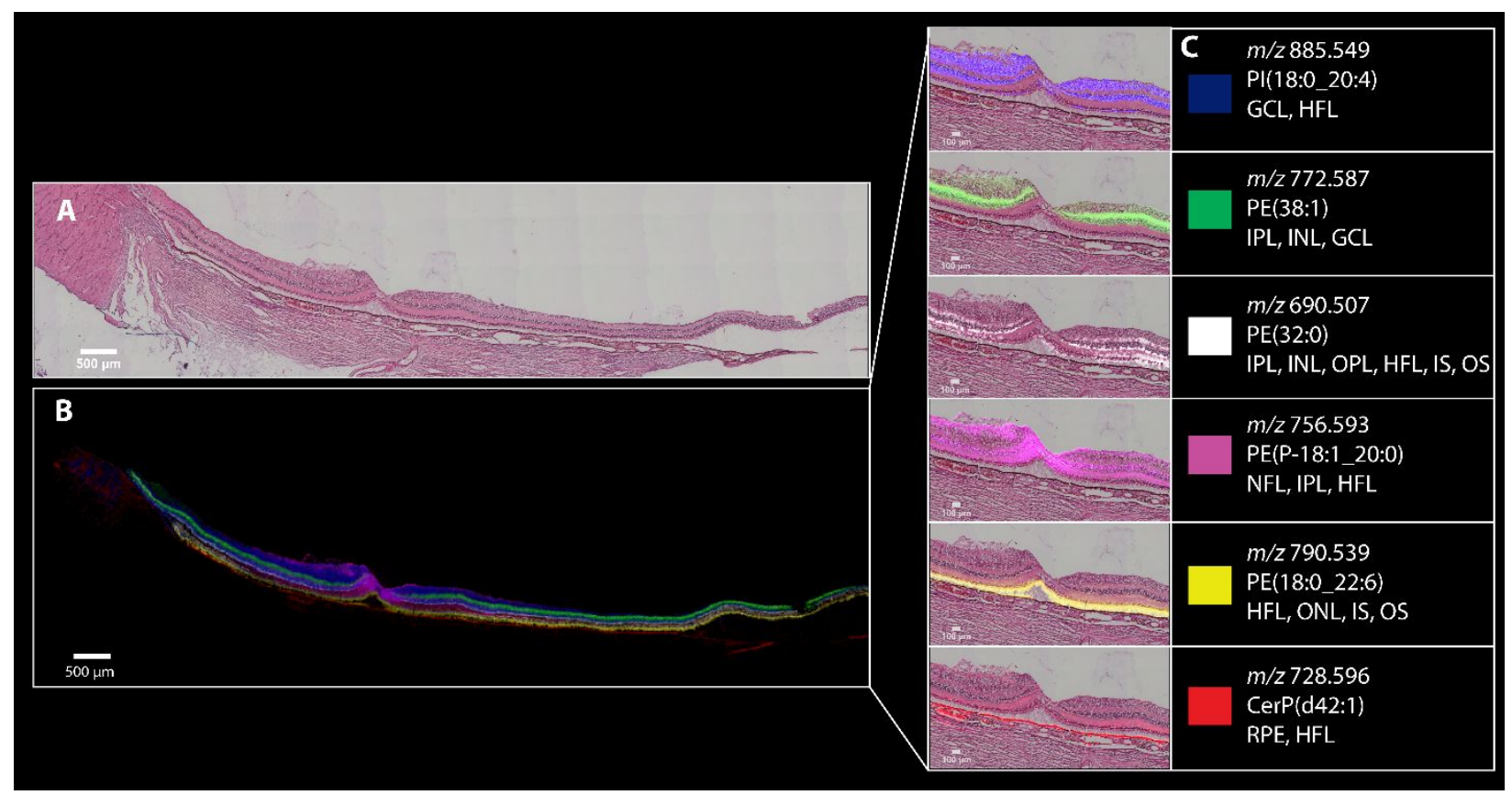

Supplemental Figure 2. Biologic replicate of data in Figure 2 showing 6 distinct signals in human macula. H\&E and MALDI IMS images of ocular tissue from an 81-year-old donor displaying six signals with distinct cellular localizations. A) Un-zoomed H\&E stained tissue. B) Overlay of MALDI IMS images showing selected lipid signals that localize to distinct layers of the neural retina (10 $\mu \mathrm{m}$ pixel size). C) Individual ion signals overlaid on top of H\&E stained tissue image. 


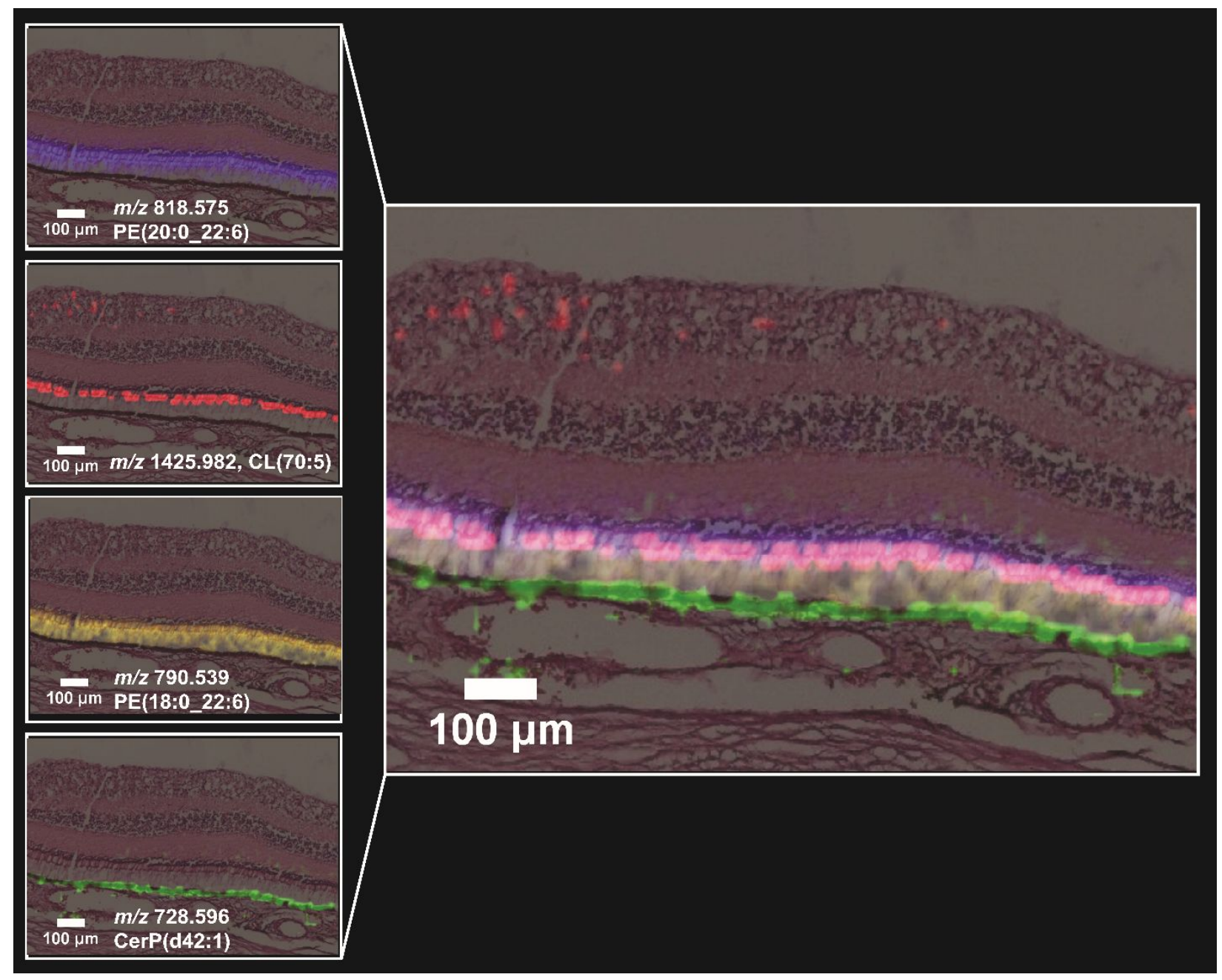

Supplemental Figure 3. Biologic replicate of imaging mass spectrometry signals in photoreceptors shown in Figure 3. MALDI IMS images and H\&E stained tissue images from 81 year old donor overlaid in peripheral retina displaying signals that localize to subcellular compartments of outer retinal cells ( $15 \mu \mathrm{m}$ pixel size). Unlike the images in Figure 3 and Supplemental 5 , and like those in Figure 2, the intense signal for $m / z 728.596$ (green) localizes to a thick layer possibly representing localization in cell bodies of RPE in addition to apical processes. These differences in localization may be due to the attachment of RPE to photoreceptors, the lower resolution of scanning in this figure, or both. 

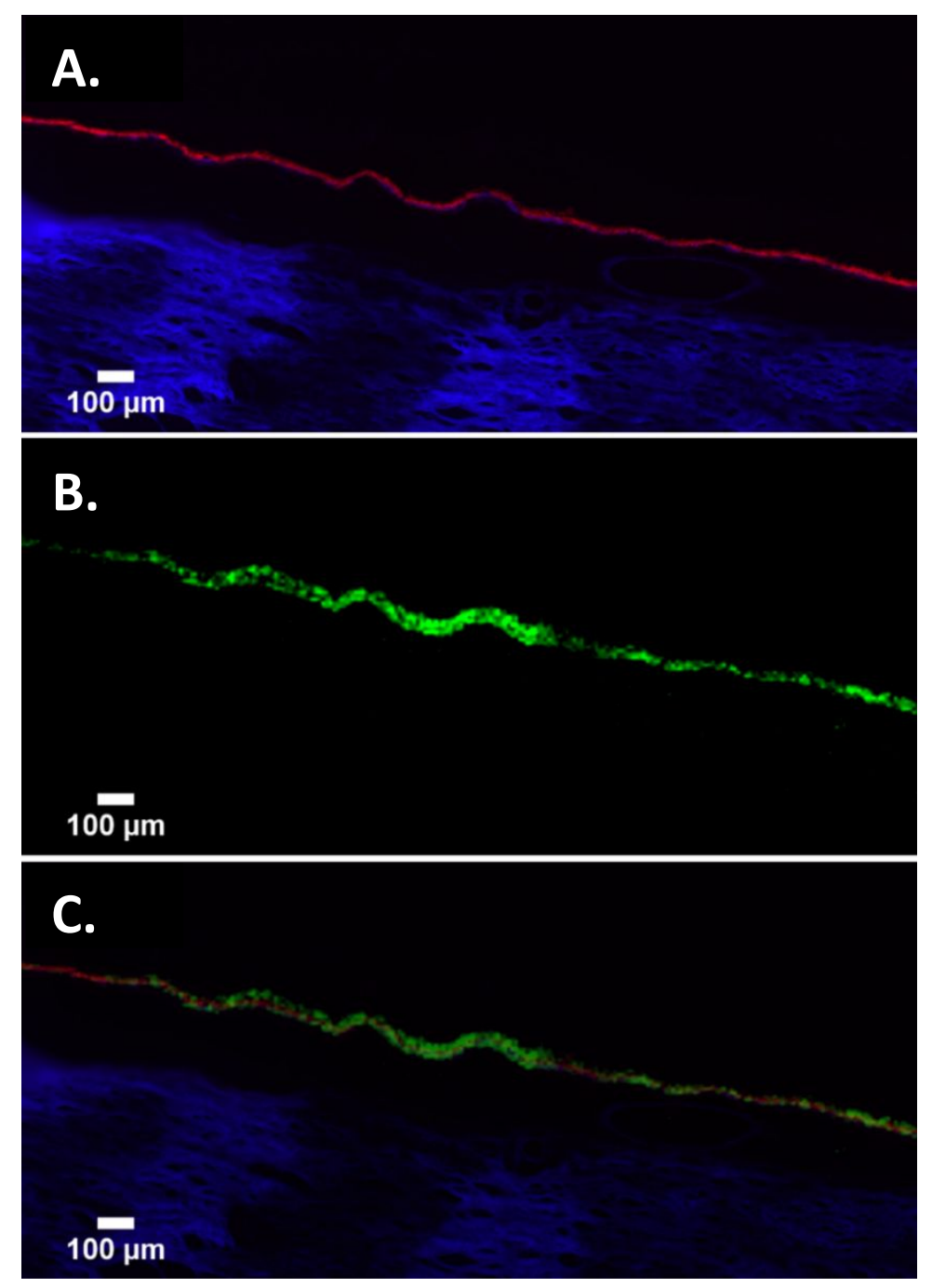

Supplemental Figure 4. Microscopy assists imaging mass spectrometry localization. A) Autofluorescence (AF) imaging of tissue from a 83 year old donor displaying intense signal originating from RPE (red) and sclera (blue). The choroid (between RPE and sclera) is not visible. B) MALDI IMS of $\mathrm{m} / \mathrm{z} 728.596$ (10 $\mu \mathrm{m}$ pixel size). C) AF overlaid with $\mathrm{m} / \mathrm{z} 728.596$ in green. 

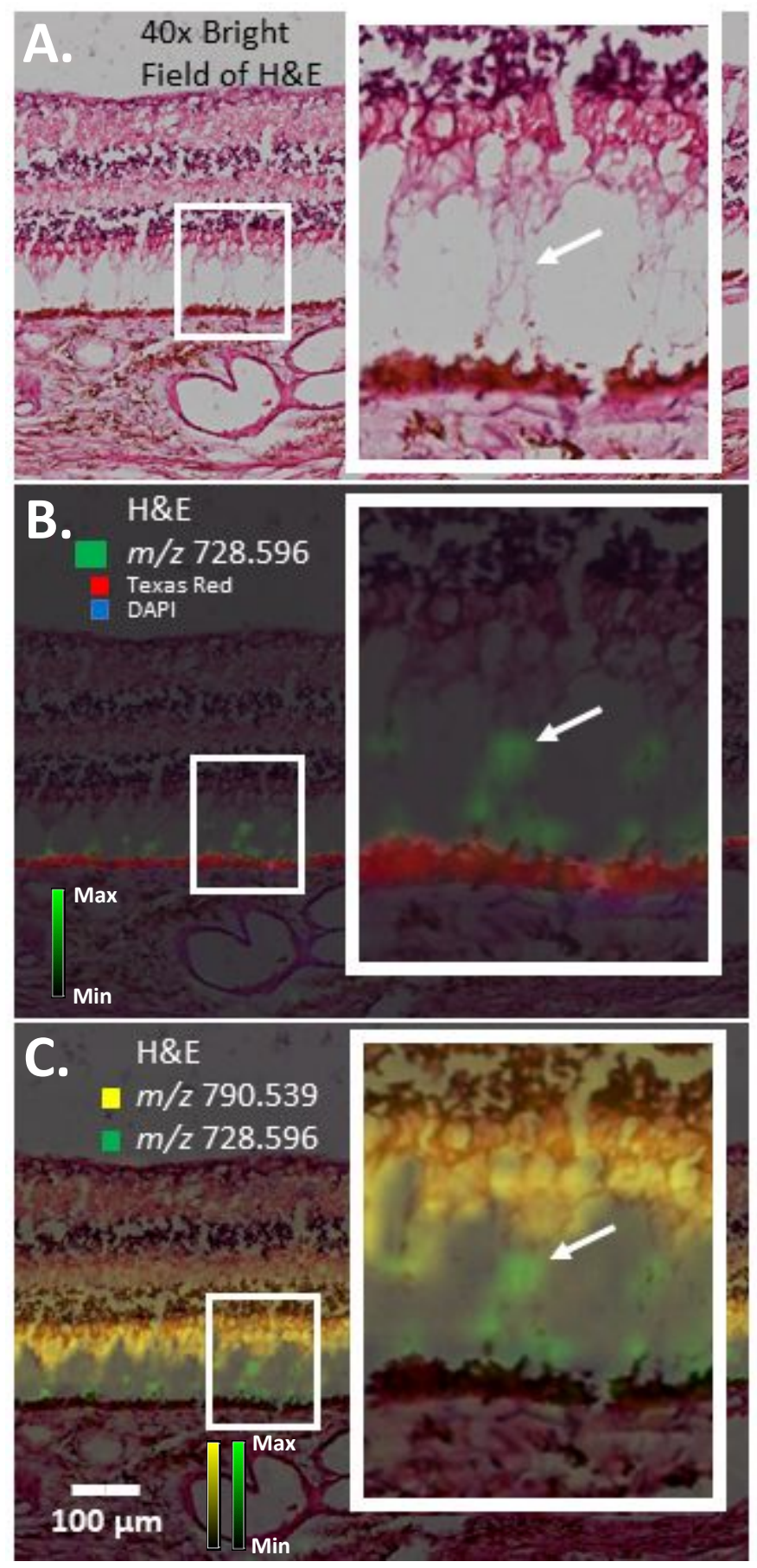

Supplemental Figure 5. Evidence for molecular localization to RPE apical processes. A) A 40X H\&E stained tissue displaying a region where the photoreceptors have separated artifactually from the RPE. B) 10x H\&E stained tissue overlaid with AF and MALDI IMS of $\mathrm{m} / \mathrm{z}$ 728.596 CerP(d42:1), captured at $10 \mu \mathrm{m}$ resolution. Image insets include arrows indicating a tissue region where this signal is present outside the RPE cell bodies, which can be seen in the overlay image in panel B inset. C) $10 x$ H\&E stained tissue overlaid with MALDI IMS of $\mathrm{m} / \mathrm{z}$ 728.596 CerP(d42:1) and $\mathrm{m} / \mathrm{z}$ 790.539 PE(18:0_22:6). Inset with arrow indicates clear separation between the 2 signals. 


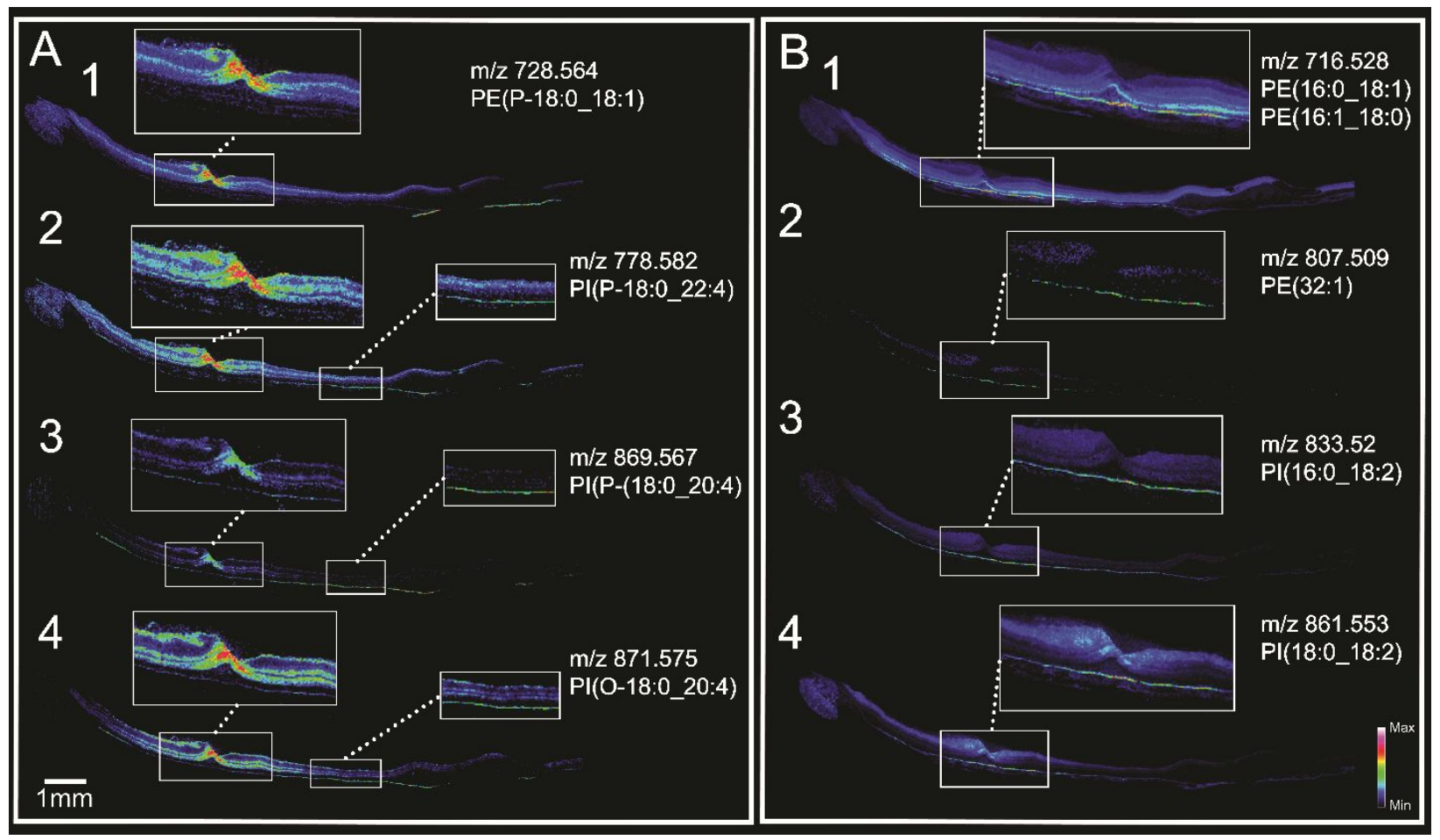

Supplemental Figure 6. MALDI IMS images of complementary macular and RPE signals from an 81-year-old donor (15 $\mu \mathrm{m}$ pixel size). Biologic replicate of data shown in main Figure 4. A) Signals localized to layers of the macular neurosensory retina and peripheral RPE. B) Signals localized to RPE underlying the macula and not confined to the fovea. 


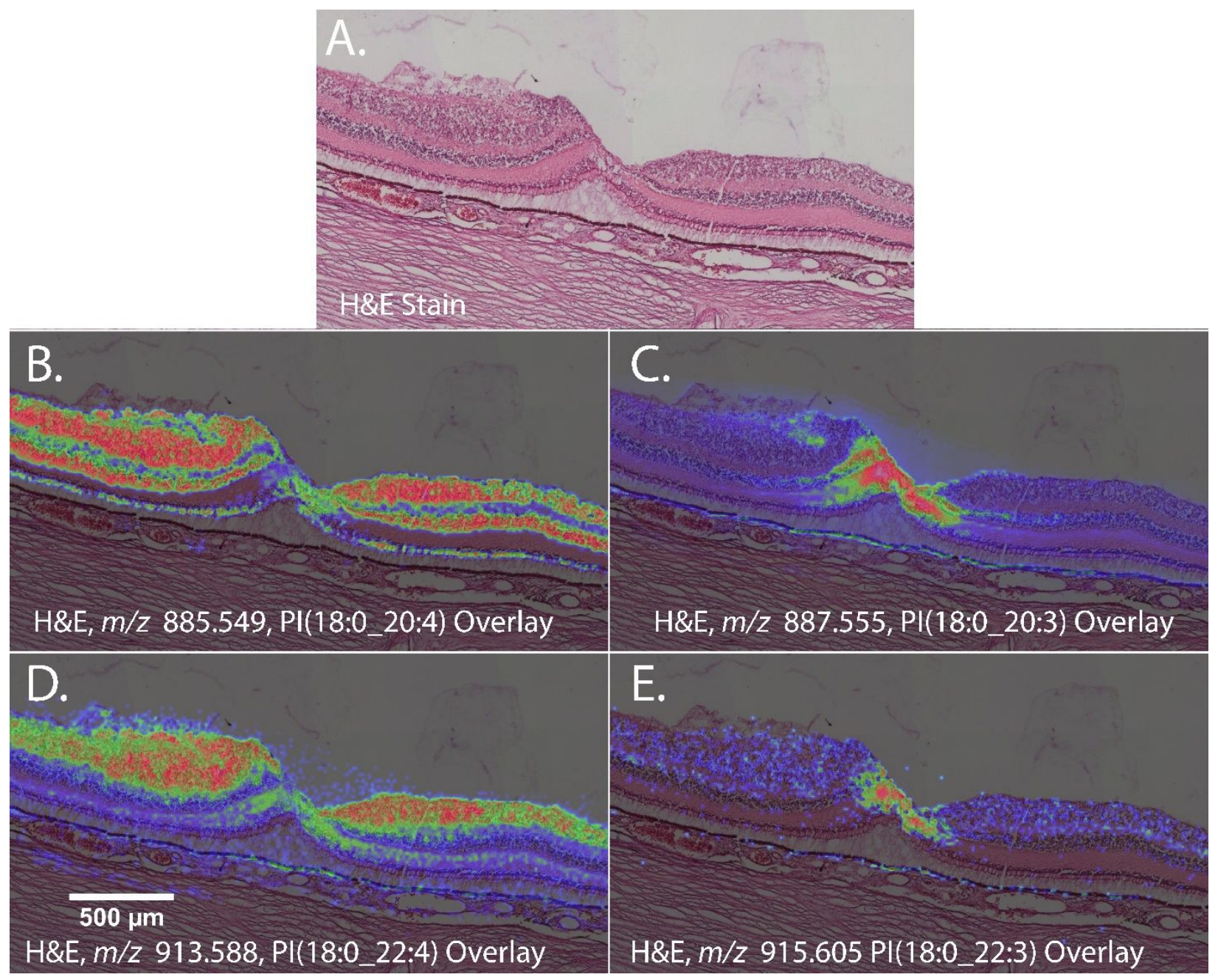

Supplemental Figure 7. Lipid species varying in only one double bond can exhibit different MALDI IMS distributions. Biologic replicate of data shown in main Figure 5. A) Zoomed H\&E stained tissue image of the fovea within the macula. B) $\mathrm{m} / \mathrm{z} 885.549$, $\mathrm{PI}\left(18: 0 \_20: 4\right)$. C) $m / z$ 887.555, PI(18:0_20:3). D) $m / z$ 913.588, PI(18:0_22:4). E) $m / z$ 915.605, $\mathrm{PI}\left(18: 0 \_22: 3\right)$. 


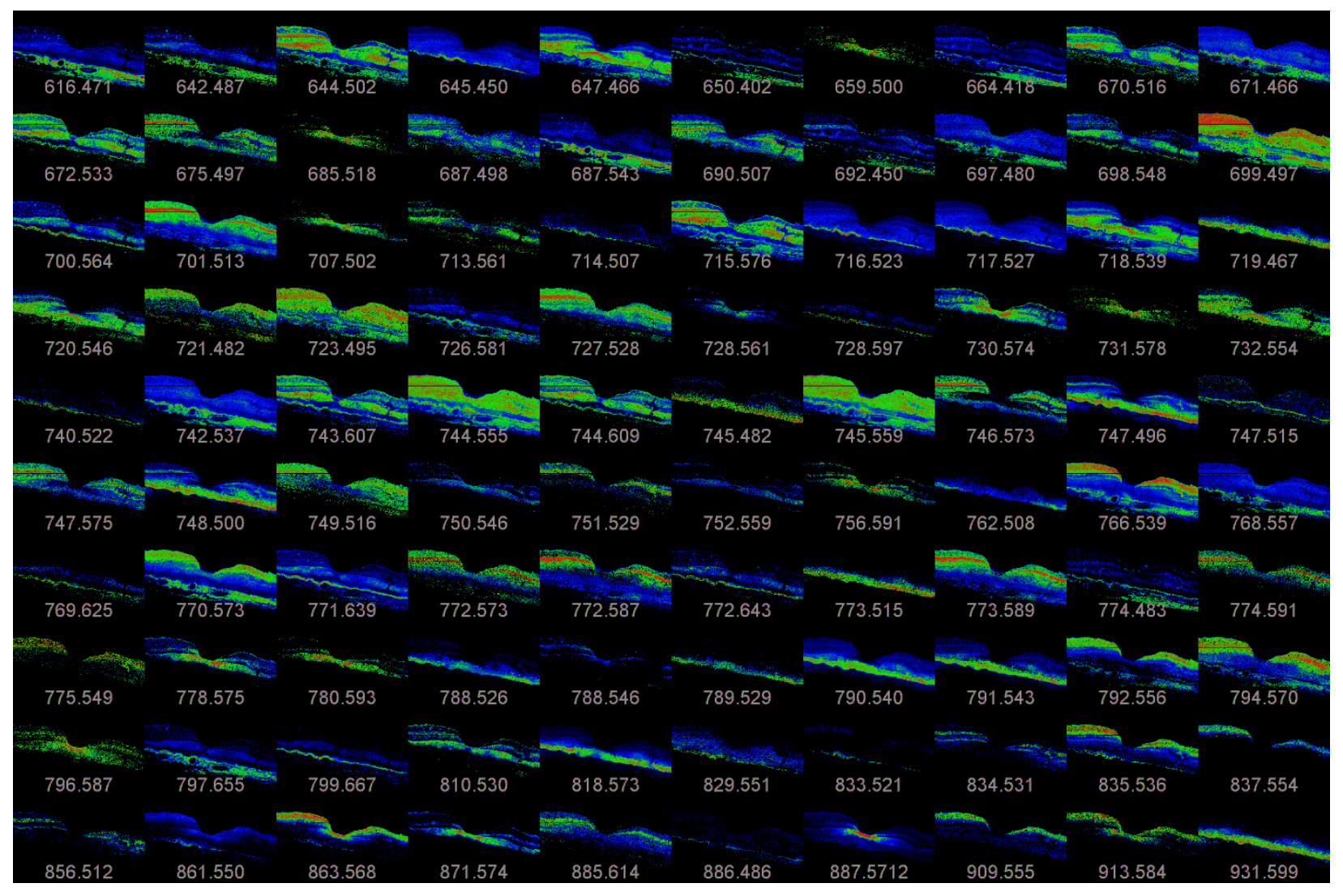

Supplemental Figure 8. Extensive dataset obtained by imaging mass spectrometry of retina and choroid in an 83-year-old human eye donor. From a dataset of 486 negative ion mode MALDI IMS images, 100 with qualitatively strong signals and varied localizations are shown. The macula, with foveal dip in the center, is flanked by perifovea. 


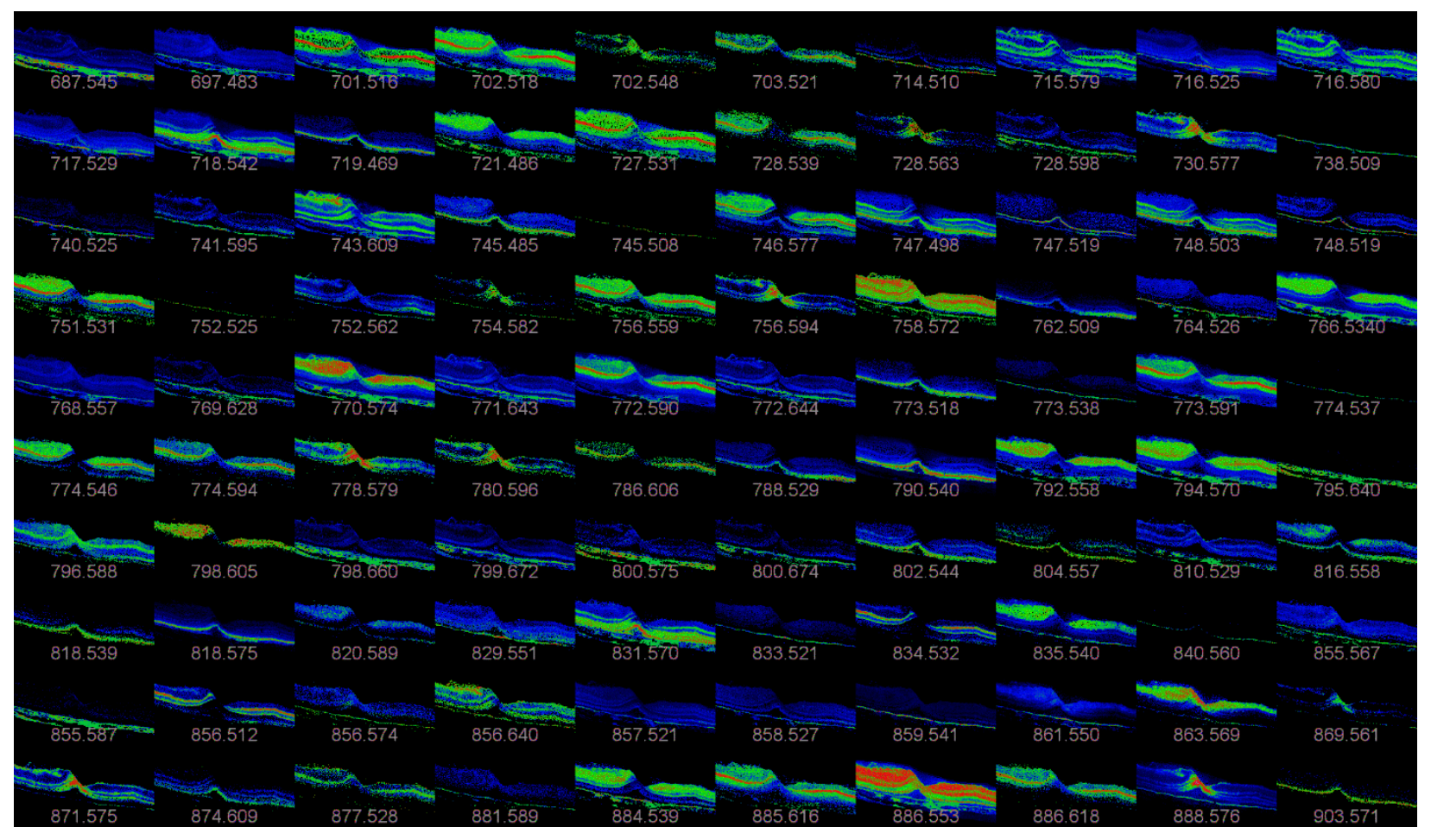

Supplemental Figure 9. Extensive dataset obtained by imaging mass spectrometry of retina and choroid in an 81-year-old human eye donor. From a dataset of 1,209 negative ion mode MALDI IMS images, 100 with qualitatively strong signals and varied localizations are shown. The macula, with foveal dip in the center, is flanked by perifovea. 
Supplemental Table 1. Tandem LC-MS/MS identifications from the 81-year-old donor tissue and accurate mass identification of $\mathrm{m} / \mathrm{z}$ values from figures. 
Tandem MS Identifications

\begin{tabular}{|c|c|c|c|c|}
\hline $\mathrm{m} / \mathrm{z}$ & ID & Th $\mathrm{m} / \mathrm{z}$ & ppm & MF \\
\hline 253.2172 & {$[16: 1-H]-$} & 253.2168 & -1.58 & C16H29O2- \\
\hline 255.2328 & {$[16: 0-H]-$} & 255.2324 & -1.57 & $\mathrm{C} 16 \mathrm{H} 3102-$ \\
\hline 281.2487 & {$[18: 1-\mathrm{H}]-$} & 281.2481 & -2.13 & $\mathrm{C} 18 \mathrm{H} 33 \mathrm{O} 2-$ \\
\hline 283.2655 & {$[18: 0-\mathrm{H}]-$} & 283.2637 & -6.35 & $\mathrm{C} 18 \mathrm{H} 35 \mathrm{O} 2-$ \\
\hline 478.2942 & Loss of $s n 2$ acyl chain as ketene $(R C H=C=O)$, $[M-(16 ; 0)-H]-$ & 478.2934 & -1.67 & C23H45NO7P- \\
\hline 480.3116 & Loss of $s n 2$ acyl chain as ketene $(R C H=C=O)$, [M- $(16: 1)-H]-$ & 480.3091 & -5.20 & C23H47NO7P- \\
\hline 460.2818 & {$[M-(16: 0)-H]-$} & 460.2828 & 2.17 & C23H43NO6P- \\
\hline 462.3 & {$[M-(16: 1)-H]-$} & 462.2985 & -3.24 & $\mathrm{C} 23 \mathrm{H} 45 \mathrm{NO} 6 \mathrm{P}-$ \\
\hline 716.5228 & $\mathrm{M}-\mathrm{H}-$ & 716.5231 & 0.42 & C39H75NO8P- \\
\hline 716.526 & MALDI MASS & & 4.47 & ppm error MALDILC \\
\hline 716.5228 & LC MS MASS & & & \\
\hline \multicolumn{5}{|c|}{$\operatorname{PE}\left(\mathrm{P}-18: 0 \_18: 1\right), \mathrm{m} / \mathrm{z} 728.563$} \\
\hline $\mathrm{m} / \mathrm{z}$ & ID & Th $\mathrm{m} / \mathrm{z}$ & ppm & $\mathrm{MF}$ \\
\hline 140.0109 & Head group & 140.0114 & 3.57 & C2H7NO4P- \\
\hline 281.2487 & {$[18: 0-\mathrm{H}]-$} & 281.2481 & -2.13 & $\mathrm{C} 18 \mathrm{H} 35 \mathrm{O} 2-$ \\
\hline 446.3026 & {$[M-(18: 1)-H]-$} & 446.3036 & 2.24 & C23H45NO5P- \\
\hline 728.5624 & $\mathrm{M}-\mathrm{H}-$ & 728.56 & -3.29 & C41H79NO3P- \\
\hline 728.563 & MALDI MASS & & & ppm error MALDILC \\
\hline 728.5624 & LC MS MASS & & 0.82 & Measurments \\
\hline \multicolumn{5}{|c|}{$\mathrm{PE}\left(\mathrm{P}-20: 0 \_18: 1\right), \mathrm{m} / \mathrm{z} 756.593$} \\
\hline $\mathrm{m} / \mathrm{z}$ & ID & Th $\mathbf{m} / \mathbf{z}$ & ppm & MF \\
\hline 281.2487 & {$[18: 1-\mathrm{H}]-$} & 281.2481 & -2.13 & $\mathrm{C} 18 \mathrm{H} 33 \mathrm{O} 2-$ \\
\hline 474.3359 & {$[M-(18: 1)-H]-$} & 474.3349 & -2.11 & C25H49NO5P- \\
\hline 492.3481 & Loss of $s n 2$ acyl chain as ketene $(R C H=C=O)$, $[M-(18: 1)-H]-$ & 492.3454 & -5.48 & C25H51NO6P- \\
\hline 756.5947 & M-H- & 756.5913 & -4.49 & C43H84NO7P- \\
\hline 756.5930 & MALDI MASS & & & ppm error MALDILC \\
\hline 756.5947 & LC MS MASS & & -2.25 & Measurments \\
\hline \multicolumn{5}{|c|}{$\mathrm{PE}\left(\mathrm{P}-18: 0 \_22: 4\right), \mathrm{m} / \mathrm{z} 778.574$} \\
\hline $\mathrm{m} / \mathrm{z}$ & ID & Th $\mathbf{m} / \mathbf{z}$ & ppm & MF \\
\hline 331.2644 & {$[22: 4-H]-$} & 331.2637 & -2.11 & $\mathrm{C} 22 \mathrm{H} 35 \mathrm{O} 2-$ \\
\hline 464.3144 & Loss of $s n 2$ acyl chain as ketene $(R C H=C=O)$, [M- $(22: 4)-H]-$ & 464.314 & -0.86 & C23H47NO6P- \\
\hline 778.5765 & $\mathrm{M}-\mathrm{H}-$ & 778.5751 & -1.80 & C45H81NO7P- \\
\hline 778.574 & MALDI MASS & & -323 & ppm error MALDILC \\
\hline 778.5765 & LC MS MASS & & -3.21 & Measurments \\
\hline 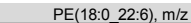 & & & & \\
\hline $\mathrm{m} / \mathrm{z}$ & ID & Th $\mathbf{m} / \mathbf{z}$ & ppm & MF \\
\hline 283.2665 & {$[18: 0-\mathrm{H}]-$} & 283.2637 & -9.88 & $\mathrm{C} 18 \mathrm{H} 3502-$ \\
\hline 327.2331 & {$[22: 6-\mathrm{H}]-$} & 327.2324 & -2.14 & $\mathrm{C} 22 \mathrm{H} 31 \mathrm{O} 2-$ \\
\hline 462.2997 & {$[\mathrm{M}-(22: 6)-\mathrm{H}]-$} & 462.2985 & -2.60 & $\mathrm{C} 23 \mathrm{H} 45 \mathrm{NO} 6 \mathrm{P}-$ \\
\hline 480.3080 & Loss of sn2 acyl chain as ketene $(\mathrm{RCH}=\mathrm{C}=\mathrm{O})$, [M-(22:6)-H]- & 480.3091 & 2.29 & C23H47NO7P- \\
\hline 790.5378 & $\mathrm{M}-\mathrm{H}-$ & 790.5387 & 1.14 & C45H77NO8P- \\
\hline 790.539 & MALDI MASS & & & ppm error MALDILC \\
\hline 790.5378 & LC MS MASS & & 1.52 & Measurments \\
\hline $\mathrm{PE}(20: 0 \quad 22: 6), \mathrm{m} / \mathrm{z}$ & & & & \\
\hline $\mathrm{m} / \mathrm{z}$ & ID & Th $\mathrm{m} / \mathbf{z}$ & ppm & $\mathrm{MF}$ \\
\hline 327.2332 & {$[22: 6-\mathrm{H}]-$} & 327.2324 & -2.44 & $\mathrm{C} 22 \mathrm{H} 3102-$ \\
\hline 490.3319 & {$[M-(22: 6)-H]-$} & 490.3297 & -4.49 & C25H49NO6P- \\
\hline 508.3408 & Loss of $s n 2$ acyl chain as ketene $(\mathrm{RCH}=\mathrm{C}=0)$, [M- $(22: 6)-\mathrm{H}]-$ & 508.3404 & -0.79 & C25H51NO7P- \\
\hline 818.5727 & 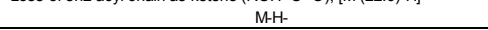 & 818.5705 & -2.69 & C47H82NO8P- \\
\hline 818.575 & MALDI MASS & & 281 & ppm error MALDILC \\
\hline 818.5727 & LC MS MASS & & 2.81 & Measurments \\
\hline $\mathrm{PI}\left(16: 0 \_18: 2\right) / \mathrm{PI}\left(16: 1 \_18: 1\right.$ & & & & \\
\hline $\mathrm{m} / \mathbf{z}$ & ID & Th $\mathrm{m} / \mathrm{z}$ & ppm & MF \\
\hline 255.2333 & {$[16: 0-H]-$} & 255.2324 & -3.53 & $\mathrm{C} 16 \mathrm{H} 3102-$ \\
\hline 279.2327 & {$[(18: 2-\mathrm{H}]-$} & 279.2324 & -1.07 & $\mathrm{C} 18 \mathrm{H} 3102-$ \\
\hline 577.2809 & {$[M-(16: 0)-H]-$} & 577.2778 & -5.37 & $\mathrm{C} 27 \mathrm{H} 46 \mathrm{O} 11 \mathrm{P}-$ \\
\hline 553.2782 & {$[\mathrm{M}-(18: 2)-\mathrm{H}]-$} & 553.2778 & -0.72 & $\mathrm{C} 25 \mathrm{H} 46 \mathrm{O} 11 \mathrm{P}-$ \\
\hline 833.5195 & $\mathrm{M}-\mathrm{H}-$ & 833.5181 & -1.68 & C43H78013P- \\
\hline 833.523 & MALDI MASS & & 420 & ppm error MALDILC \\
\hline 833.5195 & LC MS MASS & & & Measurments \\
\hline $\mathrm{Pl}\left(16: 0 \_20: 4\right), \mathrm{m} / \mathrm{z}$ & & & & \\
\hline $\mathrm{m} / \mathbf{z}$ & ID & Th $\mathbf{m} / \mathbf{z}$ & ppm & MF \\
\hline 255.2333 & {$[16: 0-\mathrm{H}]-$} & 255.2324 & -3.53 & $\mathrm{C} 16 \mathrm{H} 3102-$ \\
\hline 303.2327 & {$[20: 4-H]-$} & 303.2324 & -0.99 & $\mathrm{C} 2 \mathrm{OH} 31 \mathrm{O} 2-$ \\
\hline 601.2759 & {$[M-(16: 0)-H]-$} & 601.2778 & 3.16 & $\mathrm{C} 29 \mathrm{H} 46011 \mathrm{P}-$ \\
\hline 553.2786 & {$[M-(20: 4)-H]-$} & 553.2778 & -1.45 & $\mathrm{C} 25 \mathrm{H} 46011 \mathrm{P}-$ \\
\hline 857.5206 & $\mathrm{M}-\mathrm{H}-$ & 857.5181 & -2.92 & $\mathrm{C} 45 \mathrm{H} 78 \mathrm{O} 13 \mathrm{P}-$ \\
\hline 857.52 & MALDI MASS & & -0.699691646 & ppm error MALDI LC \\
\hline 857.5206 & LC MS MASS & & -0.699691646 & Measurments \\
\hline $\mathrm{PI}(18: 0$ 18:2), $\mathrm{m} / \mathrm{z}$ & & & & \\
\hline $\mathrm{m} / \mathbf{z}$ & ID & Th $m / z$ & ppm & $\mathrm{MF}$ \\
\hline 279.2329 & {$[(18: 2-H]-$} & 279.2324 & -1.79 & $\mathrm{C} 18 \mathrm{H} 31 \mathrm{O} 2-$ \\
\hline 283.2654 & {$[18: 0-\mathrm{H}]-$} & 283.2637 & -6.00 & C18H35O2- \\
\hline 579.2933 & {$[M-(18: 0)-H]-$} & 579.2935 & 0.35 & $\mathrm{C} 27 \mathrm{H} 48 \mathrm{O} 11 \mathrm{P}-$ \\
\hline 581.3095 & {$[\mathrm{M}-(18: 2)-\mathrm{H}]-$} & 581.3091 & -0.69 & C27H50011P- \\
\hline 861.5482 & $\mathrm{M}-\mathrm{H}-$ & 861.5494 & 1.39 & C45H82O13P- \\
\hline 861.5520 & MALDI MASS & & & ppm error MALDILC \\
\hline 861.5482 & LC MS MASS & & 4.41 & Measurments \\
\hline $\mathrm{Pl}(\mathrm{P}-18: 020: 4), \mathrm{m} / \mathrm{z}$ & & & & \\
\hline $\mathrm{m} / \mathrm{z}$ & ID & Th $\mathrm{m} / \mathbf{z}$ & ppm & MF \\
\hline 241.0116 & Head group & 241.0114 & -0.83 & $\mathrm{C} 6 \mathrm{H} 1008 \mathrm{P}-$ \\
\hline 303.2331 & {$[20: 4-H]-$} & 303.2324 & -2.31 & $\mathrm{C} 2 \mathrm{OH} 31 \mathrm{O} 2-$ \\
\hline 403.2621 & {$[M-(20: 4)-164-H]-$} & 403.2614 & -1.74 & $\mathrm{C} 21 \mathrm{H} 40 \mathrm{OP}$ - \\
\hline 565.3174 & {$[M-(20: 4)-H]-$} & 565.3142 & -5.66 & $\mathrm{C} 27 \mathrm{H} 50010 \mathrm{P}-$ \\
\hline 869.5566 & $\mathrm{M}-\mathrm{H}-\mathrm{-}$ & 869.5549 & -1.96 & C47H82O12P- \\
\hline 869.555 & MALDI MASS & & -1.96 & ppm error MALDILC \\
\hline 869.5566 & LC MS MASS & & & Measurments \\
\hline
\end{tabular}




\begin{tabular}{|c|c|c|c|c|}
\hline $\mathrm{Pl}\left(\mathrm{O}-18: 0 \_20: 4\right), \mathrm{m} / \mathrm{z}$ & & & & \\
\hline $\mathrm{m} / \mathbf{z}$ & ID & Th $\mathrm{m} / \mathbf{z}$ & ppm & MF \\
\hline 303.233 & {$[20: 4-H]-$} & 303.2324 & -1.98 & $\mathrm{C} 2 \mathrm{OH} 3102-$ \\
\hline 405.2778 & {$[M-(20: 4)-164-H]-$} & 405.277 & -1.97 & $\mathrm{C} 21 \mathrm{H} 42 \mathrm{OP} \mathrm{P}-$ \\
\hline 567.3306 & {$[\mathrm{M}-(20: 4)-\mathrm{H}]-$} & 567.3299 & -1.23 & C27H52O10P- \\
\hline 871.572 & $\mathrm{M}-\mathrm{H}-\mathrm{H}$ & 871.5701 & -2.18 & C47H84O12P- \\
\hline 871.574 & MALDI MASS & & 222 & ppm error MALDI LC \\
\hline 871.572 & LC MS MASS & & 2.29 & Measurments \\
\hline \multicolumn{5}{|c|}{$\mathrm{PI}(18: 0 \quad 20: 4), \mathrm{m} / \mathrm{z} 885.549$} \\
\hline $\mathrm{m} / \mathrm{z}$ & ID & Th $\mathrm{m} / \mathbf{z}$ & ppm & MF \\
\hline 283.2644 & {$[18: 0-\mathrm{H}]-$} & 283.2637 & -2.47 & $\mathrm{C} 18 \mathrm{H} 35 \mathrm{O} 2-$ \\
\hline 303.2331 & {$[20: 4-H]-$} & 303.2324 & -2.31 & $\mathrm{C} 2 \mathrm{OH} 3102-$ \\
\hline 419.2575 & Neutral loss of sn2 RCOOH group and inositol from [M-164-(20:4)]- & 419.2562 & -3.10 & $\mathrm{C} 21 \mathrm{H} 4006 \mathrm{P}-$ \\
\hline 581.3107 & {$[\mathrm{M}-(20: 4)-\mathrm{H}]-$} & 581.3091 & -2.75 & $\mathrm{C} 27 \mathrm{H} 50011 \mathrm{P}-$ \\
\hline 599.3188 & Loss of sn2 acyl chain as ketene $(\mathrm{RCH}=\mathrm{C}=\mathrm{O})$, [M-(20:4)-H]- & 599.3201 & 2.17 & C27H52O12P- \\
\hline 885.5518 & M-H- & 885.5488 & -3.39 & C47H83O13P- \\
\hline 885.549 & MALDI MASS & & & ppm error MALDI LC \\
\hline 885.5518 & LC MS MASS & & -3.16 & Measurments \\
\hline \multicolumn{5}{|c|}{$\mathrm{PI}\left(18: 0 \_20: 3\right), \mathrm{m} / \mathrm{z} 887.567$} \\
\hline $\mathrm{m} / \mathrm{z}$ & ID & Th $\mathrm{m} / \mathbf{z}$ & ppm & MF \\
\hline 241.0117 & $\mathrm{M}-\mathrm{H}-\mathrm{-}$ & 241.0114 & -1.24 & $\mathrm{C} 6 \mathrm{H} 1008 \mathrm{P}-$ \\
\hline 283.2645 & {$[18: 0-H]-$} & 283.2643 & $\begin{array}{l}-1.24 \\
-0.71\end{array}$ & $\mathrm{C} 18 \mathrm{H} 35 \mathrm{O} 2-$ \\
\hline 305.2487 & {$[20: 3-\mathrm{H}]-$} & 305.2481 & -1.97 & $\mathrm{C} 2 \mathrm{OH} 33 \mathrm{O} 2-$ \\
\hline 419.2571 & Neutral loss of sn2 RCOOH group and inositol from [M-164-(20:3)]- & 419.2563 & -1.91 & $\mathrm{C} 21 \mathrm{H} 4006 \mathrm{P}-$ \\
\hline 581.3107 & {$[\mathrm{M}-(20: 3)-\mathrm{H}]-$} & 581.3091 & -2.75 & C27H50011P- \\
\hline 887.5656 & $\mathrm{M}-\mathrm{H}-\mathrm{H}^{-}$ & 887.565 & -0.68 & C47H84O13P- \\
\hline 887.567 & MALDI MASS & & 158 & ppm error MALDI LC \\
\hline 887.5656 & LC MS MASS & & 1.58 & Measurments \\
\hline \multicolumn{5}{|c|}{$\mathrm{Pl}(18: 0 \quad 22: 4), \mathrm{m} / \mathrm{z} 913.587$} \\
\hline $\mathrm{m} / \mathrm{z}$ & ID & Th $\mathrm{m} / \mathbf{z}$ & ppm & MF \\
\hline 241.0116 & Head group & 241.0114 & -0.83 & $\mathrm{C} 6 \mathrm{H} 1008 \mathrm{P}$ \\
\hline 283.2643 & {$[18: 0-\mathrm{H}]-$} & 283.2643 & 0.00 & $\mathrm{C} 18 \mathrm{H} 35 \mathrm{O} 2-$ \\
\hline 331.2643 & {$[22: 4-\mathrm{H}]-$} & 331.2643 & 0.00 & $\mathrm{C} 22 \mathrm{H} 36 \mathrm{O} 2$ \\
\hline 419.2575 & Neutral loss of sn2 RCOOH group and inositol from [M-164-(22:4)]- & 419.2563 & -2.86 & $\mathrm{C} 21 \mathrm{H} 40 \mathrm{O} 6 \mathrm{P}-$ \\
\hline 581.3099 & [M-(22:4)-H]- & 581.3091 & -1.38 & $\mathrm{C} 27 \mathrm{H} 50011 \mathrm{P}$ \\
\hline 913.5828 & $\mathrm{M}-\mathrm{H}-\mathrm{T}$ & 913.5812 & -1.75 & C47H82O12P- \\
\hline 913.587 & MALDI MASS & & 175 & ppm error MALDILC \\
\hline 913.5828 & LC MS MASS & & -1.75 & Measurments \\
\hline \multicolumn{5}{|c|}{$\mathrm{PI}(18: 0$ 22:3), $\mathrm{m} / \mathrm{z} 915.601$} \\
\hline$\frac{\mathrm{Pl}(18: 022: 3), \mathrm{m} / \mathrm{z} \mathrm{C}}{\mathrm{m} / \mathbf{z}}$ & ID & Th $\mathrm{m} / \mathbf{z}$ & ppm & MF \\
\hline 241.0112 & Head group & 241.0114 & 0.83 & $\mathrm{C} 6 \mathrm{H} 10 \mathrm{OP} \mathrm{P}$ \\
\hline 283.2655 & {$[18: 0-\mathrm{H}]-$} & 283.2643 & -4.24 & $\mathrm{C} 18 \mathrm{H} 35 \mathrm{O} 2-$ \\
\hline 333.2792 & {$[22: 3-\mathrm{H}]-$} & 333.2799 & 2.10 & $\mathrm{C} 22 \mathrm{H} 37 \mathrm{O} 2-$ \\
\hline 419.2585 & Neutral loss of sn2 RCOOH group and inositol from [M-164-(22:3)]- & 419.2563 & -5.25 & $\mathrm{C} 21 \mathrm{H} 40 \mathrm{O} 6 \mathrm{P}$ \\
\hline 581.3055 & [M-(22:3)-H]- & 581.3091 & 6.19 & $\mathrm{C} 27 \mathrm{H} 50011 \mathrm{P}-$ \\
\hline 915.6005 & $\mathrm{M}-\mathrm{H}-\mathrm{-}$ & 915.5963 & -4.59 & C49H88O13P- \\
\hline 915.601 & MALDI MASS & & 459 & ppm error MALDI LC \\
\hline 915.6005 & LC MS MASS & & -4.59 & Measurments \\
\hline \multicolumn{5}{|c|}{ MALDI Accurate Mass Identifications } \\
\hline \multicolumn{5}{|c|}{$\operatorname{CerP}(\mathrm{d} 42: 1), \mathrm{m} / \mathrm{z} 728.596$} \\
\hline $\mathrm{m} / \mathbf{z}$ & ID & Th $\mathbf{m} / \mathbf{z}$ & ppm & MF \\
\hline 728.596 & {$[\operatorname{CerP}(\mathrm{d} 42: 1)-\mathrm{H}]-$} & 728.5964 & 0.55 & $\mathrm{C} 42 \mathrm{H} 84 \mathrm{NO} 6 \mathrm{P}-\mathrm{-}$ \\
\hline \multicolumn{5}{|c|}{$\mathrm{CL}(70: 5), \mathrm{m} / \mathrm{z} 1425.9818$} \\
\hline $\mathrm{m} / \mathbf{z}$ & ID & Th $m / z$ & ppm & $\mathrm{MF}$ \\
\hline 1425.9818 & {$[\mathrm{CL}(70: 5)-\mathrm{H}]-$} & 1425.9801 & -1.19 & $\begin{array}{ll}\mathrm{C} 79 \mathrm{H} 143017 \mathrm{P} 2- \\
\end{array}$ \\
\hline \multicolumn{5}{|c|}{$\mathrm{PE}(38: 1), \mathrm{m} / \mathrm{z} 772.5874$} \\
\hline$\frac{\mathrm{m} / \mathrm{z}}{\mathrm{z}}$ & ID & Th $\mathrm{m} / \mathbf{z}$ & ppm & MF \\
\hline 772.587 & {$[\mathrm{PE}(38: 1)-\mathrm{H}]-$} & 772.5862 & -1.04 & C43H84NO8P- \\
\hline \multicolumn{5}{|c|}{$\mathrm{PI}(32: 1), \mathrm{m} / \mathrm{z} 807.501$} \\
\hline $\mathrm{m} / \mathbf{z}$ & ID & Th $\mathbf{m} / \mathbf{z}$ & ppm & MF \\
\hline 807.501 & {$[P(32: 1)-H]-$} & 807.5024 & 1.73 & C41H76013P- \\
\hline \multicolumn{5}{|c|}{$\mathrm{PE}(32: 0), \mathrm{m} / \mathrm{z} 690.507$} \\
\hline $\mathrm{m} / \mathbf{z}$ & ID & Th $\mathrm{m} / \mathbf{z}$ & ppm & MF \\
\hline 690.507 & {$[\mathrm{PE}(32: 0)-\mathrm{H}]-$} & 690.5074 & 0.58 & C37H73NO8P- \\
\hline
\end{tabular}


Supplemental Tables 2. Top ten signal ion intensities from each layer in the neural retina.

\begin{tabular}{|c|c|c|}
\hline \multicolumn{3}{|c|}{ RPE } \\
\hline $\mathbf{m} / \mathbf{z}$ & Intensity & Relative Intensity \\
\hline 744.5546 & 1928685 & 100.0 \\
716.5236 & 1577054 & 81.8 \\
885.5498 & 1228665 & 63.7 \\
673.4815 & 849318 & 44.0 \\
687.5429 & 759467 & 39.4 \\
790.5398 & 623279 & 32.3 \\
771.6396 & 555966 & 28.8 \\
645.4499 & 542415 & 28.1 \\
797.6540 & 447064 & 23.2 \\
766.5385 & 419039 & 21.7 \\
699.4975 & 389918 & 20.2 \\
\hline
\end{tabular}

\begin{tabular}{|c|c|c|}
\hline \multicolumn{3}{|c|}{ Photoreceptors } \\
\hline $\mathbf{m} / \mathbf{z}$ & Intensity & Relative Intensity \\
\hline 885.5484 & 4091437 & 100.0 \\
790.5395 & 3316202 & 81.1 \\
718.5396 & 2536730 & 62.0 \\
744.5547 & 1691615 & 41.3 \\
818.5730 & 1552271 & 37.9 \\
747.4954 & 1409496 & 34.4 \\
673.4816 & 940251 & 23.0 \\
647.4662 & 790063 & 19.3 \\
931.5969 & 482744 & 11.8 \\
772.5879 & 476546 & 11.6 \\
766.5385 & 439487 & 10.7 \\
\hline
\end{tabular}

\begin{tabular}{|c|c|c|}
\hline \multicolumn{3}{|c|}{ Henle Fiber Layer } \\
\hline $\mathbf{m} / \mathbf{z}$ & Intensity & Relative Intensity \\
\hline 885.5486 & 7138865 & 100.0 \\
744.5538 & 5101973 & 71.5 \\
673.4811 & 2144893 & 30.0 \\
718.5391 & 1806065 & 25.3 \\
718.5391 & 1806065 & 25.3 \\
715.5762 & 1463977 & 20.5 \\
772.5873 & 1169007 & 16.4 \\
699.4973 & 1130907 & 15.8 \\
699.4973 & 1130907 & 15.8 \\
790.5392 & 976084 & 13.7 \\
687.5425 & 933322 & 13.1 \\
\hline
\end{tabular}

ONL 


\begin{tabular}{|c|c|c|}
\hline $\mathbf{m} / \mathbf{z}$ & Intensity & Relative Intensity \\
\hline 885.5492 & 5873040 & 100.0 \\
718.5398 & 3525832 & 60.0 \\
744.5551 & 3012916 & 51.3 \\
790.5405 & 2975648 & 50.7 \\
818.5742 & 1500630 & 25.6 \\
673.4811 & 1468254 & 25.0 \\
747.4958 & 1376379 & 23.4 \\
647.4655 & 1063291 & 18.1 \\
716.5240 & 1011430 & 17.2 \\
715.5770 & 993406 & 16.9 \\
772.5887 & 810386 & 13.8 \\
\hline
\end{tabular}

\begin{tabular}{|c|c|c|}
\hline \multicolumn{3}{|c|}{ OPL } \\
\hline $\mathbf{m} / \mathbf{z}$ & Intensity & Relative Intensity \\
\hline 885.5485 & 6174113 & 100.0 \\
744.5544 & 4877430 & 79.0 \\
718.5394 & 3674836 & 59.5 \\
673.4816 & 1990568 & 32.2 \\
772.5877 & 1520859 & 24.6 \\
715.5767 & 1398235 & 22.6 \\
790.5396 & 1206185 & 19.5 \\
647.4666 & 1114809 & 18.1 \\
699.4978 & 890434 & 14.4 \\
701.5136 & 869758 & 14.1 \\
742.5372 & 813733 & 13.2 \\
\hline
\end{tabular}

\begin{tabular}{|c|c|c|}
\hline \multicolumn{3}{|c|}{ INL } \\
\hline $\mathbf{m} / \mathbf{z}$ & Intensity & Relative Intensity \\
\hline 885.5490 & 5586378 & 100.0 \\
744.5545 & 3894083 & 69.7 \\
772.5877 & 1506124 & 27.0 \\
772.5877 & 1506124 & 27.0 \\
718.5397 & 1488353 & 26.6 \\
673.4818 & 1450697 & 26.0 \\
715.5768 & 1095446 & 19.6 \\
701.5137 & 834948 & 14.9 \\
699.4978 & 773287 & 13.8 \\
770.5722 & 729120 & 13.1 \\
790.5397 & 684883 & 12.3 \\
\hline
\end{tabular}




\begin{tabular}{|c|c|c|}
\hline $\mathbf{m} / \mathbf{z}$ & Intensity & Relative Intensity \\
\hline 885.5486 & 5779566 & 100.0 \\
744.5544 & 4467074 & 77.3 \\
772.5876 & 2378774 & 41.2 \\
673.4812 & 1838084 & 31.8 \\
718.5395 & 1650464 & 28.6 \\
715.5765 & 1428622 & 24.7 \\
701.5133 & 1413709 & 24.5 \\
766.5383 & 1262327 & 21.8 \\
699.4974 & 1104369 & 19.1 \\
770.5721 & 1067286 & 18.5 \\
794.5693 & 876961 & 15.2 \\
\hline
\end{tabular}

\begin{tabular}{|c|c|c|}
\hline \multicolumn{3}{|c|}{ NFL } \\
\hline $\mathbf{m} / \mathbf{z}$ & Intensity & Relative Intensity \\
\hline 885.5482 & 9493662 & 100.0 \\
744.5543 & 4381912 & 46.2 \\
766.5383 & 2235294 & 23.5 \\
673.4811 & 1742639 & 18.4 \\
770.5720 & 1694981 & 17.9 \\
772.5880 & 1522012 & 16.0 \\
699.4974 & 1316910 & 13.9 \\
794.5694 & 1056590 & 11.1 \\
718.5396 & 968629 & 10.2 \\
701.5134 & 912853 & 9.6 \\
701.5134 & 912853 & 9.6 \\
\hline
\end{tabular}

\begin{tabular}{|c|c|c|}
\hline \multicolumn{3}{|c|}{ GCL } \\
\hline $\mathbf{m} / \mathbf{z}$ & Intensity & Relative Intensity \\
\hline 885.5497 & 3288517 & 100 \\
744.5545 & 2020274 & 61.4 \\
766.5386 & 927160 & 28.2 \\
673.4814 & 854309 & 26.0 \\
673.4814 & 854309 & 26.0 \\
770.5722 & 695147 & 21.1 \\
772.5881 & 667077 & 20.3 \\
699.4974 & 617317 & 18.8 \\
715.5766 & 470877 & 14.3 \\
701.5135 & 466973 & 14.2 \\
718.5396 & 435619 & 13.2 \\
\hline
\end{tabular}

